\title{
Selection effects in identifying magnetic clouds and the importance of the closest approach parameter
}

\author{
R. P. Lepping ${ }^{1}$ and C.-C. $\mathrm{Wu}^{2}$ \\ ${ }^{1}$ Heliosphysics Science Division, NASA-Goddard Space Flight Center, Greenbelt, MD 20771, USA \\ ${ }^{2}$ Navel Research Laboratory, Washington, D.C. 20735, USA
}

Received: 10 December 2009 - Revised: 2 August 2010 - Accepted: 5 August 2010 - Published: 18 August 2010

\begin{abstract}
This study is motivated by the unusually low number of magnetic clouds (MCs) that are strictly identified within interplanetary coronal mass ejections (ICMEs), as observed at $1 \mathrm{AU}$; this is usually estimated to be around $30 \%$ or lower. But a looser definition of MCs may significantly increase this percentage. Another motivation is the unexpected shape of the occurrence distribution of the observers' "closest approach distances" (measured from a MC's axis, and called CA) which drops off somewhat rapidly as $|\mathrm{CA}|$ (in \% of MC radius) approaches $100 \%$, based on earlier studies. We suggest, for various geometrical and physical reasons, that the $|\mathrm{CA}|$-distribution should be somewhere between a uniform one and the one actually observed, and therefore the $30 \%$ estimate should be higher. So we ask, When there is a failure to identify a MC within an ICME, is it occasionally due to a large $|\mathrm{CA}|$ passage, making MC identification more difficult, i.e., is it due to an event selection effect? In attempting to answer this question we examine WIND data to obtain an accurate distribution of the number of MCs vs. $|\mathrm{CA}|$ distance, whether the event is ICME-related or not, where initially a large number of cases $(N=98)$ are considered. This gives a frequence distribution that is far from uniform, confirming earlier studies. This along with the fact that there are many ICME identification-parameters that do not depend on $|\mathrm{CA}|$ suggest that, indeed an $M C$ event selection effect may explain at least part of the low ratio of (No. MCs)/(No. ICMEs). We also show that there is an acceptable geometrical and physical consistency in the relationships for both average "normalized" magnetic field intensity change and field direction change vs. $|\mathrm{CA}|$ within a $\mathrm{MC}$, suggesting that our estimates of $|\mathrm{CA}|, B_{O}$ (magnetic field intensity on the axis), and choice of a proper "cloud coordinate" system (all needed in the analysis) are acceptably accurate. Therefore,
\end{abstract}

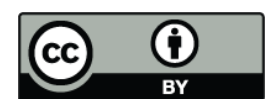

Correspondence to: C.-C. Wu (chin-chun.wu@nrl.navy.mil) the MC fitting model (Lepping et al., 1990) is adequate, on average, for our analysis. However, this selection effect is not likely to completely answer our original question, on the unexpected ratio of MCs to ICMEs, so we must look for other factors, such as peculiarities of CME birth conditions. As a by-product of this analysis, we determine that the first order structural effects within a MC due to its interaction with the solar wind, plus the MC's usual expansion at $1 \mathrm{AU}$ (i.e., the non-force free components of the MC's field) are, on average, weakly dependent on radial distance from the MC's axis; that is, in the outer reaches of a typical MC the non-force free effects show up, but even there they are rather weak. Finally, we show that it is not likely that a MC's size distribution statistically controls the occurrence distribution of the estimated $|\mathrm{CA}| \mathrm{s}$.

Keywords. Interplanetary physics (Interplanetary magnetic fields; Solar wind plasma)

\section{Introduction}

A magnetic cloud (MC) was originally defined empirically in terms of in-situ spacecraft measurements of magnetic fields and particles in the interplanetary medium, viz., it is a region in the solar wind having: (1) an enhanced magnetic field strength, (2) a smooth change in field direction as observed by a spacecraft passing through the MC, and (3) a low proton temperature (and low proton plasma beta) compared to the ambient proton temperature (Burlaga et al., 1981, 1990; Klein and Burlaga, 1982; Burlaga, 1995). MCs are also understood tacitly to be large structures averaging about $20 \mathrm{~h}$ in duration at $1 \mathrm{AU}$ for the better examples (e.g., Lepping et al., 2006). MCs have been observed at distances other than at $1 \mathrm{AU}$, for example by the Helios spacecraft (e.g., Bothmer and Schwenn, 1998) and the Voyager spacecraft (e.g., Skoug et al., 2000; Burlaga et al., 2001). MCs have been revealed

Published by Copernicus Publications on behalf of the European Geosciences Union. 
in general to be large magnetic flux ropes in the solar wind, i.e., plasma embedded relatively strong magnetic fields of approximately helical structure (e.g., Marubashi, 1986, 1997: Priest, 1990; Gosling, 1990; Lepping et al., 1990; Burlaga, 1995; Kumar and Rust, 1996).

An interplanetary coronal mass ejection (ICME) is an interplanetary manifestation of a coronal mass ejection (CME), which is strictly a solar event and well described by Schwenn (1996) and Gosling (1997); also see a comprehensive study of ICMEs by Richardson and Cane (2004) and Gopalswamy (2006). ICMEs are usually identified by their differences from the solar wind by some subset of about twelve potentially applicable quantities, including the same quantities used for MCs (e.g., enhanced magnetic field intensity, smoothly changing field direction, and relatively low proton temperature (e.g., Gosling et al., 1973) and low proton plasma beta), as well as by bidirectional streaming of electrons (e.g., Bame et al., 1981), bidirectional streaming of low energy protons (e.g., Marsden et al., 1987), high charge states of ions and compositional signatures, e.g., examination of Mg10+/O6+ (e.g., Henke et al.,1998, 2001), low charge states (e.g., Zwickl et al., 1982; Galvin 1997; Burlaga et al., 1998; Gopalswamy et al., 1998), singly charged $\mathrm{He}^{+}$(e.g., Schwenn et al., 1980), and bidirectional particle flows at cosmic ray energies $(\sim 1 \mathrm{MeV})$ (e.g., Richardson et al., 2000), and bidirectional solar wind electron heat flux events (BDEs) (e.g., Gosling et al., 1990), and including ground based data, the occurrence of a one or two step Forbush decrease (Forbush, 1937; Cane, 2000; Cane and Lario, 2006). Also see Schwenn (1996) who points out the important fact that of the many potential signatures of interplanetary plasma clouds (meaning ICMEs, in our terminology) usually only a subset actually occurs for any given case. And see Russell and Shinde (2005) and Russell et al. (2005), who developed a derived parameter to define ICMEs which considers the combined plasma and field pressure perpendicular to the local magnetic field; this method strives to make ICME identification more unique.

Both kinds of interplanetary structures (MCs and ICMEs), and others generally listed under the rubric of "plasma clouds," where all of which often move faster than the normal solar wind speed in their vicinity (at least at $1 \mathrm{AU}$ ), have been referred to as solar ejecta or just ejecta (e.g., Burlaga, 1995). Ejecta are important because: (1) they always contain useful information about their dramatic birth conditions at the Sun (e.g., Webb et al., 2001; Gopalswamy et al., 2008), (2) they often drive interplanetary shocks which accelerate charged particles (e.g., Lee and Fisk, 1982; Kahler, 2001), and (3) portions of their extent, even their upstream sheath regions, are often markedly geoeffective (e.g., Burlaga et al., 1981; McComas et al., 1988; McPherron, 1995; Wu et al., 2006), because of generally having long-lasting strong southward magnetic fields and the usually higher than background solar wind speeds. Also see Luhmann (1997) who discusses the importance of CMEs to Space Weather generally.
This study attempts to address the issue of why there are not more MCs observed within ICMEs as observed at $1 \mathrm{AU}$ (e.g., for an early example of a MC associated with a coronal mass ejection see Burlaga et al., 1982). For example, it has been asserted that only 10-30\% of MCs are observed within ICMEs (Gosling, 1990). Recent studies by Richardson and Cane (2010) and Wu and Lepping (2010) agree with the upper limit of this range, although there is not complete agreement on this percentage in the space science community at large, e.g. see Xu et al. (2009); also see Richardson and Cane (2004). We suggest that the percentage of ICMEs containing MCs should be higher, perhaps much higher, because an ICME is an interplanetary manifestation of a CME, and such an event is typically the result of a large explosive event on the Sun of twisted magnetic field lines (usually from an erupting prominence) and so also is a MC (Bothmer and Schwenn, 1994; Webb et al., 2000; Gopalswamy, 2006). And the explosion itself is due to a magnetic process, although the details of which are not fully agreed on. Consistently, MCs depend on strong interplanetary magnetic fields for an essential part of their identification and so also do ICMEs, or they usually do. Hence, it is surprising that the occurrence of these two types of events are not more highly correlated. There may be several explanations. First, we point out that the definition of a MC being quite strict and that for a ICME being much less so is obviously part of the discrepancy in the occurrence rate of these two entities, and we speculate that if any magnetic structure even remotely resembling a $\mathrm{MC}$ were accepted as a real MC (e.g., accepting the greater number of magnetic cloud-like (MCL) objects found by Lepping et al., 2005, in WIND data), then the number of MCs would obviously increase dramatically. This may be an important, and perhaps the main, explanation for the discrepancy, especially since ICMEs correlate better with the combination of MCs and MCLs than with just bone fide MCs (see, e.g., Wu and Lepping, 2010). But below we suggest other explanations.

When there is this "failure" to identify an existing MC within an ICME, is it at least occasionally due to a large closest approach (CA) passage of the spacecraft from the MC's axis, making the MC more difficult to identify, i.e., is it due to a selection effect? Or is it a natural consequence of CME birth conditions, in the sense that apparently some (or many?) CMEs are simply not always born with the necessary smoothly twisted and strong magnetic field lines needed to indicate the existence of a MC? The possibility of the latter explanation on a MC's birth conditions will require study beyond our present scope, although we believe that it must be contributory for some cases. However, we may be able to shed some light on aspects of the geometrical argument, which will depend on actual and estimated $|\mathrm{CA}| \mathrm{s}$, and that will be our main focus here. (Note that we use the notation $|\mathrm{CA}|$ to indicate that we are not usually concerned with the sign of CA $\left(\equiv Y_{O} / R_{O}\right)$, which can be positive or negative, since $Y_{O}$, the "impact parameter," can be positive or negative depending on which "side" of the MC the spacecraft passes; 
$R_{O}$ is the MC's estimated radius. Also CA can mean simply "closest approach," as was used earlier, or relative closest approach distance, which is $\left|Y_{O}\right| / R_{O}$. The context will usually make the usage clear.)

It appears that this "geometrical" failure (i.e., one due to large $|\mathrm{CA}|)$ of $\mathrm{MC}$ identification is bound to occur, at least occasionally, especially for those cases where the spacecraft passes through the outer reaches of a MC where there is little change in field direction or intensity $(|\boldsymbol{B}|)$ and especially when $|\boldsymbol{B}|$ is not too different from the ambient interplanetary magnetic field. In those cases the MC would hardly be identifiable whether it is contained within an ICME or not. In this study we will, in fact, show quantitatively by using a large number of WIND MCs that there is a significant change in the variation of average field direction and average field intensity across a $\mathrm{MC}$ as the spacecraft's $|\mathrm{CA}|$ grows from zero to a large fraction of the MC's radius in a predictable way and becomes insignificantly small in both cases as $|\mathrm{CA}|$ approaches the MC's radius, as might be expected. When there is a small change especially in field direction, along the spacecraft's path within a MC, the MC is generally not going to be easily identifiable, and not likely amenable to analysis. For some of the above reasons, it is apparent that much of our analysis will require having relatively accurate estimates of $|\mathrm{CA}|$, which is a quantity that is estimated by a MC parameter fitting model and whose accuracy is expected to depend on the sophistication of the model. Part of our analysis, therefore, must show consistency among the estimated quantities of $|\mathrm{CA}|, R_{O}$ (also used as diameter, $2 R_{O}$ ), $B_{O}$ (the axial field intensity), and axial alignment, i.e., the relevant modeled quantities. Specifically, the field intensity part of the study will require the necessity to define a normalized magnetic field magnitude, and this is the reason for the use of the modeled parameter, $B_{O}$. And we must be able to find a proper model-dependent MC-coordinate system in which to do the analysis, the reason for knowledge of the axial alignment, as well as be able to estimate sufficiently accurately $|\mathrm{CA}|$ itself. We will attempt to use the estimated quantities from the Lepping et al. (1990) MC parameter fitting model for a large number of cases from the WIND mission. However, the study by Lepping et al. (2003) revealed that usually the poorest estimated quantity in the Lepping et al. (1990) model's set of seven unknowns is CA, which plays a key role in this study. Hence, it is important that we take great care to prove its adequacy for the study. Further, we attempt to show the efficacy of using the estimated quantities $B_{O}$, $R_{O}$, and $|\mathrm{CA}|$ for their true values by calculating reasonably high correlation coefficients for the comparisons of expected normalized magnetic field intensity and directional changes vs. $|\mathrm{CA}|$ through so called "consistency" arguments, as explained below.

Our specific goals using WIND magnetic field data are to examine (and interpret) the following: (1) how severely nonuniform is the distribution of $|\mathrm{CA}| \mathrm{s}$ for a large set of MCs (nonuniformity using a smaller number of cases was shown

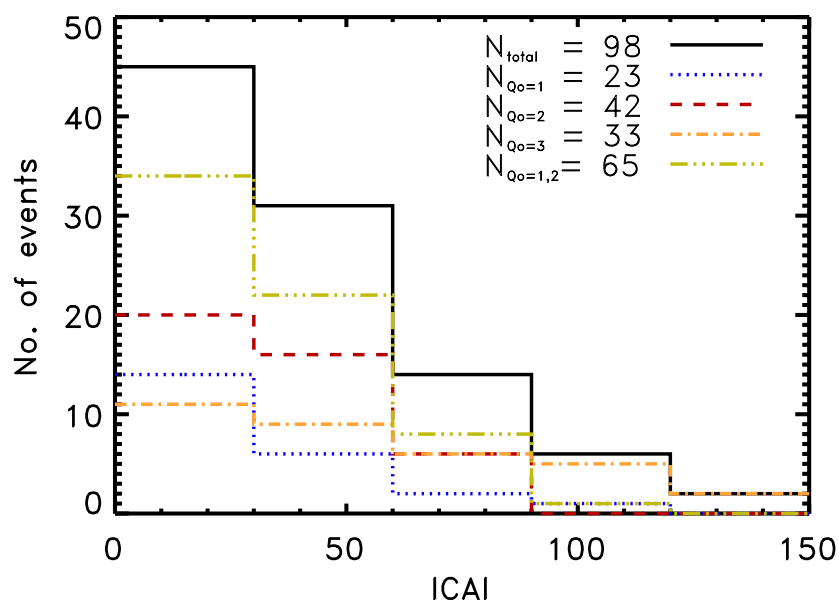

Fig. 1. Histograms of $|\mathrm{CA}|\left(\equiv\left|Y_{O}\right| / R_{O} \mid\right.$ (in \%)), for various quality $\left(Q_{O}\right)$ subsets from WIND data, over the period from early 1995 to April 2006, confirming that there is a relatively severe drop off of identified $\mathrm{MCs}$ as a function of $|\mathrm{CA}|$ distance.

preliminarily by Lepping et al., 2006), and we will argue that this severity should not be expected, unless there is a selection effect mitigating against finding cases with large $|\mathrm{CA}| \mathrm{s}$, (2) parameter "consistency arguments," described in detail below (concerning the relationships of $B_{O}, R_{O}$, and vs. $|\mathrm{CA}|)$, indeed do hold, providing useful and trustworthy estimates of these key parameters, especially $|\mathrm{CA}|$, used critically in this study, and finally (3) whether it is likely that actual MC sizes statistically control the sizes of estimated $|\mathrm{CA}| \mathrm{s}$, where all three of these questions are based on a large sample size of MCs. However we do not claim to know what the actual distribution of $|\mathrm{CA}|$ should be.

\section{The distribution of $\mathrm{MC}$ occurrences vs. $\mid \mathrm{CA}$}

We utilize the MC data from the WIND mission over the years from early 1995 to April 2006, and Table 2 on the Website http://lepmfi.gsfc.nasa.gov/mfi/mag_cloud_S1.html provides the model estimated values for $B_{O}$ and $|\mathrm{CA}|$ (called $\left|Y_{O} / R_{O}\right|$ in \%, in that table), based on the WIND magnetic field and plasma data (Lepping et al., 1995; Ogilvie et al., 1995). This table also provides Quality estimates $\left(Q_{O}=1\right.$, 2 , or 3 for excellent, good, or poor, respectively, for each of these MCs); Appendix A of Lepping et al. (2006) gives the definition of Quality used here. The MCs will be split into various sets according to this $Q_{O}$ index.

Here we show the distributions of $|\mathrm{CA}| \mathrm{s}$ for various quality sets, given in Fig. 1, i.e., the distributions are based on the full set of WIND MCs, $N=98$ and various subsets. (For the moment we assume that the accuracies of the estimated $|\mathrm{CA}| \mathrm{s}$ use here are high enough to generate a meaningful Fig. 1. In Sects. 3.0 and 4.0 we defend this belief.) First, we point out that although $|\mathrm{CA}|$ (in \%) should in principle go from 0 to 


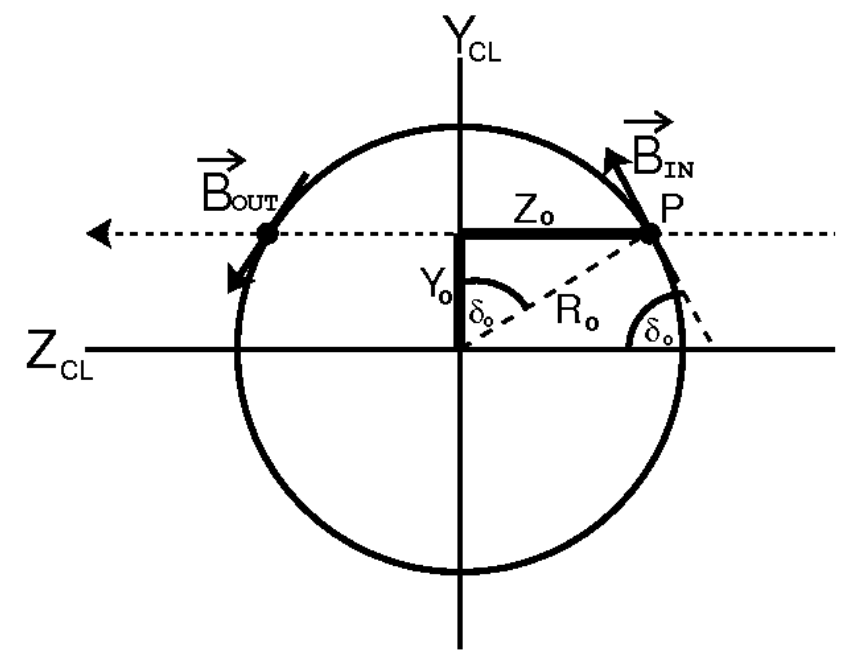

Fig. 2. The ideal cross-section of a magnetic cloud showing a spacecraft passing through whose projected path is parallel to the $Z_{\mathrm{CL}}$ axis in cloud (CL) coordinates (see text) with a closest approach distance $Y_{O}$. The $\boldsymbol{X}_{\mathrm{CL}}$ axis (normal to the plane of the figure) is parallel to the cloud's axis and co-located.

$100 \%$, we see that the distribution in Fig. 1 exceeds $100 \%$ for the $Q_{O}=3$ set and hence also for the full set. This is simply due to the fact that there is uncertainty in the estimations of the $|\mathrm{CA}| \mathrm{s}$ for all sets (being especially poor for the $Q_{O}=3$ set and even more so for the few cases where $|\mathrm{CA}|$ is near $100 \%)$, as discussed by Lepping et al. (2003, 2004), and in the model there is no constraint that $Y_{O}$ must be equal to or less than $R_{O}$. But it is clear that in all cases we are considering actual MCs, as has been discussed elsewhere (Lepping et al., 2006; and in this same paper a preliminary version of Fig. 1 is shown using a smaller number of MCs), so the actual $|\mathrm{CA}|$ must be less than $100 \%$, except for special and rare cases discussed in the Summary and Conclusions where this overall issue is more extensively discussed. Therefore, the few cases where $|\mathrm{CA}|$ was erroneously estimated to be $>100 \%$ by the Lepping et al. (1990) MC fitting routine, they are in actuality $|\mathrm{CA}|<100 \%$ and usually are very near $100 \%$, but we cannot give the exact actual value of $|\mathrm{CA}|$, of course.

We see in Fig. 1 that there is a somewhat dramatic drop of numbers of events as $|\mathrm{CA}|$ gets larger, for all subsets, becoming for the $N=98$ set, for example, only a small fraction of the total at $|\mathrm{CA}|=90 \%$. A complete and accurate distribution of actual MCs may not be exactly uniform, as we discuss in the Appendix A, but all of those in Fig. 1 seem to be unrealistic and probably are due mainly to a selection effect as we get closer to a $|\mathrm{CA}|$ of $100 \%$. In Fig. 1 we show each subset separately, because, even though the full set is apparently adequate for the argument, it has many poorly estimated $|\mathrm{CA}| \mathrm{s}$ (mainly from the incorporated $Q_{O}=3$ set). But, even though the good sets, especially the $Q_{O}=1$ set, give more trustworthy results, there are far fewer cases $(N=23)$. In any case, all sets support the same basic conclusion: the distribution is markedly different from uniform. But the statistical accuracy of the estimates of the parameter $|\mathrm{CA}|$ used here is key to any conclusions drawn, of course. The following addresses that issue.

\section{Analysis: the "consistency arguments"}

Here we develop formulations for examining the variation in the magnetic field direction and intensity $(|\boldsymbol{B}|)$ vs. $|\mathrm{CA}|$, separately, using the MCs with combined Quality of $Q=1,2$ which provides 65 events. The main purpose here is to show that on average the model-estimated quantities $R_{O}$ and $|\mathrm{CA}|$ are accurate enough for our analysis. There is usually little uncertainty in $R_{O}$. We will start by examining the change in observed field direction just within the inbound and outbound boundaries for a set of MCs vs. $|\mathrm{CA}|$.

\subsection{Angle $\alpha$ vs. $|\mathrm{CA}|$}

We define an angle related to the maximum magnetic field directional change across the $\mathrm{MC}$ for a given $|\mathrm{CA}|$, after irrelevant field "noise" is reduced by field averaging. For this purpose it is useful to define a so-called magnetic cloud (CL) coordinate system, where $\boldsymbol{X}_{\mathrm{CL}}$ is the unit vector along the MC's axis, positive along the direction of the magnetic field at the axis, $\boldsymbol{Y}_{\mathrm{CL}}$ is in the MC's cross-sectional plane along the closest approach vector, and $\boldsymbol{X}_{\mathrm{CL}} \times \boldsymbol{Y}_{\mathrm{CL}}=\boldsymbol{Z}_{\mathrm{CL}}$; notice that $\boldsymbol{Z}_{\mathrm{CL}}$ is the projection of the spacecraft's trajectory in the MC's cross-section, positive toward the Sun. The means of obtaining the transformation matrix to go from geocentric solar ecliptic (GSE) coordinates (in which the magnetic field data originates in our case) to CL coordinates, as well as to see the final formulation of the matrix itself in terms of $\theta_{A}$ and $\phi_{A}$ (which are the estimated latitude and longitude, respectively, of the MC's axis in the GSE system) is given at the Website with URL http://lepmfi.gsfc.nasa.gov/mfi/ecliptic.html.

The two angles $\theta_{A}$ and $\phi_{A}$ uniquely define the CL coordinate system and have been estimated by the Lepping et al. (1990) MC fitting model for our analysis. Then we define an angle $\alpha$, which is ideally expected to be that between the magnetic field just inside the MC's inbound boundary $\left(\boldsymbol{B}_{\mathrm{IN}}\right)$ and that just inside the outbound boundary $\left(\boldsymbol{B}_{\text {OUT }}\right)$, as projected into the MC's cross-sectional plane (the $\boldsymbol{Y}_{\mathrm{CL}^{-}}$ $Z_{\mathrm{CL}}$ plane as seen in Fig. 2), by the following:

$\alpha=\cos ^{-1}\left(\boldsymbol{B}_{\mathrm{IN}} /\left|B_{\mathrm{IN}}\right| \times \boldsymbol{B}_{\text {OUT }} /\left|B_{\mathrm{OUT}}\right|\right)_{\mathrm{CL}}$.

We will use various interval averaging-lengths for these fields to help reduce field fluctuations that are considered "noise" in the sense that the fluctuations are not considered part of the underlying MC structure. The angle $\delta_{O}$ is pictorially defined in Fig. 2. We claim that $\alpha \approx 2 \delta_{O}$, where $\alpha$ is defined in terms of measured magnetic fields, from Eq. (1), and $\delta_{O}$ is defined in terms of what is ideally expected of the magnetic 
field just within the ideal MC's circular boundary. Notice that $\delta_{O}$ is related to only the right side, whereas $\alpha$ depends on a difference between the right boundary's slope and the left boundary's slope, as is expressed in Eq. (1), and hence from this assumed symmetry we have the factor of 2 . We then see from the expression for a circle, $Y^{2}+Z^{2}=R_{O}^{2}$, and differentiation, that

$2 Y_{O}(d Y / d Z)_{O}+2 Z_{O}=0$,

where Eq. (2) is evaluated at point $P$, and hence, we use the subscript 0 on $Y_{O}$ and $Z_{O}$. Since $(d Y / d Z)_{O}=-\tan \delta_{O}=-\sin \delta_{O} / \cos \delta_{O}$, then Eq. (2) becomes, after substitution and dividing by $-2 R_{O}$, the following

$Y_{O} / R_{O}\left(\sin \delta_{O} / \cos \delta_{O}\right)=Z_{O} / R_{O}$.

We see from Fig. 2 that ideally $\sin \delta_{O}=Z_{O} / R_{O}$, and therefore, along with the definition of "Closest Approach" $(|\mathrm{CA}|)=\left|Y_{O} /\right| / R_{O}$, Eq. (3) becomes $|\mathrm{CA}|=\cos \delta_{O}$ or, since $\alpha \approx 2 \delta_{O}$, it becomes

$|\mathrm{CA}| \approx \cos (\alpha / 2)$,

where we note that ideally $0 \leq \alpha \leq 180^{\circ}$. (Notice that by assuming symmetry we need only examine the right-hand side of Fig. 2, where for convenience we keep $Z_{O} \geq 0$.) How well Eq. (4) is satisfied depends on how well the magnetic fields near the boundaries of the MC of interest satisfy the ideal circular model, i.e., one with low noise (after averaging the field) and no appreciable MC-expansion - and on how well $R_{O}$ and $|\mathrm{CA}|$ have been estimated by the MC fitting model. Equation (4)'s validity will be checked using magnetic field data from actual MCs.

\subsection{Normalized magnitude of field vs. $|\mathrm{CA}|$}

Likewise, we examine the variation in magnetic field intensity, $|\boldsymbol{B}|$, but in the following way. For a given MC we use the estimated axial field from the Lepping et al. (1990) MC fitting model (called $B_{O}$ ), create an average of the actual field intensity $(<|\boldsymbol{B}|>)$ along the spacecraft's path, and form the ratio $<|\boldsymbol{B}|>/ B_{O}$. That is, we "normalize" the average field according to the axial field strength, where the average field value is obtained using the interval representing the spacecraft's path through the MC, i.e., the MC's encounter's duration. In this way, we try to eliminate (or at least reduce) any problem with variation in "absolute" field strength among the set of MCs and obtain a ratio that is dimensionless and that will vary only with distance from the MC's axis, i.e., with $|\mathbf{C A}|$. Since there is a large variety of average field intensities across MCs, and similarly for the $B_{O}$ s, we do not expect a good correlation for $<|\boldsymbol{B}|>$ vs. $(|\mathrm{CA}|)$ or for $B_{O}$ vs. $(|\mathrm{CA}|)$, separately. This will be tested also.

Now we ask, What do we expect for $\langle|\boldsymbol{B}|>| B_{O}$ vs. $|\mathrm{CA}|$ theoretically, if the Bessel function field of the constant $\alpha$ ' force free case for a MC used in Lepping et al. (1990) holds?
We examine this question, noting that such a model is given, in CL coordinates, by:

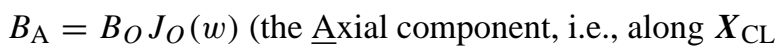
in Fig. 2)

$B_{\mathrm{T}}=B_{O} H J_{1}(w)$ (the $\underline{\text { Tangential component, i.e., }}$ perpendicular to $\mathbf{A}$ and $\mathbf{R}$ )

$B_{\mathrm{R}}=0$, (the Radial component, i.e., along $\mathbf{R}$ in Fig. 2), (5a) where

$w=\alpha^{\prime} r$,

and where $H$ is the handedness ( $H:+1$ for right-handed and -1 for left-handed); $\alpha^{\prime}$ is simply a proportionality constant that determines the pitch of the MC's field lines (and not to be confused with the angle $\alpha$ used in the analysis above). Hence, the normalize (by $B_{O}$ ) magnitude of the field is

$|B| / B_{O} \equiv f(w)$,

where

$f(w)=\sqrt{ }\left[\left(J_{O}(w)\right)^{2}+\left(J_{1}(w)\right)^{2}\right]$

from Eq. (5). We now find the average of $|\boldsymbol{B}| / B_{O}$ (called $[<|B|>\mid B o]$ theory) across the encounter-duration of the MC, based on this model. Here the average symbol $<>$ represents an average across the duration, even though we will average over only the "first half" of the encounter and assume that the results hold for the full spacecraft's encounterduration, because of symmetry. So we integrate $f(w)$ over $d Z$ from 0.0 to $Z_{O}$ (where we drop the symbol "CL" on the coordinates as being understood) along the horizontal line of the spacecraft's path as shown in Fig. 2, i.e., from the $Z=0$-point on the $\mathrm{Y}$-axis to point $\mathrm{P}$ on the boundary (where $\left.Z=Z_{O}\right)$. Hence, the average of $f(w)$ is

$\left[<|B|>/ B_{O}\right]$ theory $=\int f(w) d Z / \int d Z$,

( $Z$ integrated from 0 to $Z_{O}$; see Fig. 2),

We note that $f(w)$ is a reasonably slowly varying function of $w$, and therefore it can be extremely well fit by a cubic in $w$ :

$f(w)=A_{O}+A_{1} w+A_{2} w^{2}+A_{3} w^{3}$,

where $A_{O}=0.99720, A_{1}=0.02412, \quad A_{2}=-0.17235$, and $A_{3}=0.03296$; the sigma for the cubic fit is 0.000865 . Then we express $\left[<|B|>\mid B_{O}\right]$ theory, which we call $I$ for convenience here, as

$I=\Sigma I_{J}$ (for $J: 0$ to 3 ),

where:

$\begin{aligned} & I_{O}=\left(A_{O} / Z_{O}\right) \int d Z=A_{O}, \text { and } \\ & I_{J}=\left(A_{J} / Z_{O}\right) \int w^{J} d Z \text { (for } J: 1 \text { to } 3, \\ &\left.\text { and with integration limits of } 0 \text { to } Z_{O}\right),\end{aligned}$ 
and where $\int d Z=Z_{O}$ (in the denominator of Eq. 8), after $f(w)$ from Eq. (9) is substituted into Eq. (8). We now relate $w$ and $Z$ in the following way. First, we recognize that

$r^{2}=Y_{O}^{2}+Z^{2}$

along the spacecraft's path (i.e., at fixed $Y_{O}$ ), as shown in Fig. 2. Then, as done above, we note that specifically $Y_{O}^{2}+Z_{O}^{2}=R_{O}^{2}$, or

$Z_{O}=R_{O} \sqrt{ }\left(1-|\mathrm{CA}|^{2}\right)$,

(used for the upper limit in the integrals of Eqs. 11), and we notice that at the boundary (where $r=R_{O}$ ) $w=2.40$ (see Lepping et al., 1990), and therefore, $\alpha^{\prime}=2.40 / R_{O}$ from Eq. (5b). Hence, using these facts and considering Eqs. (5b), and (12), we obtain

$w^{2}=(2.40)^{2}\left(|\mathrm{CA}|^{2}+Z^{2} / R_{O}^{2}\right)$

Integrating Eqs. (11) using Eq. (14) yields (where again $\left[<|B|>/ B_{O}\right]$ theory $\left.\equiv I\right)$ :

$$
\begin{aligned}
I= & 0.99720+0.02895(1+K)-0.33092\left(1+2|\mathrm{CA}|^{2}\right) \\
& +0.11390\left[1+3|\mathrm{CA}|^{2}(1+K) / 2\right],
\end{aligned}
$$

where

$$
K \equiv|\mathrm{CA}|^{2}\left[\ln \left|\left(1+\sqrt{ }\left(1-|\mathrm{CA}|^{2}\right)\right) /\right| \mathrm{CA}||\right] / \sqrt{ }\left(1-|\mathrm{CA}|^{2}\right)
$$

Note that in Eqs. (15) and (16) $|\mathrm{CA}|$ ranges over 0.0 to 1.0 ; it is not in percentage. We will show below how well $I$ of Eq. (15) reproduces the actual WIND MC data for $<|B|>\mid B_{O}$ vs. $|\mathrm{CA}|$. And we stress that I was derived purely from the use of $\sqrt{ }\left[\left(J_{O}(w)\right)^{2}+\left(J_{1}(w)\right)^{2}\right]$ for the MC's magnetic field magnitude, i.e., from the force free theory. If it does not agree with real MC data, then one or all of our estimates of $|\mathrm{CA}|, B_{O}$, and the $\mathrm{CL}$ coordinate system (i.e., $\theta_{A}$ and $\phi_{A}-$ see Sect. 3.1) would be suspect, as discussed above.

\section{Results}

In calculating $<|\boldsymbol{B}|>$ or $<|\boldsymbol{B}|>\mid B_{O}$ the averages are taken over the "duration" of each spacecraft passage for each MC from the basic field data in 1-min average form. But two types of averages, i.e., of interval-lengths of $15 \mathrm{~min}$ and $1 \mathrm{~h}$, will be used for the computation of the angle $\alpha$ and $\cos (\alpha / 2)$ vs. $|\mathrm{CA}|$; this was done because we are focusing on single points just within the inbound and outbound boundaries for each MC, and such longer averages cause needed datasmoothing before computation. When all available MCs are used (i.e., for all levels of MC quality, $Q_{O}$ ) we have $N=98$ cases, but this reduces to $N=65$ cases when the poor cases $\left(Q_{O}=3\right)$ are eliminated.

In Table 1 we show what linear correlation coefficients (c.c.s) result when considering the angle $\alpha$ and $\cos (\alpha / 2)$
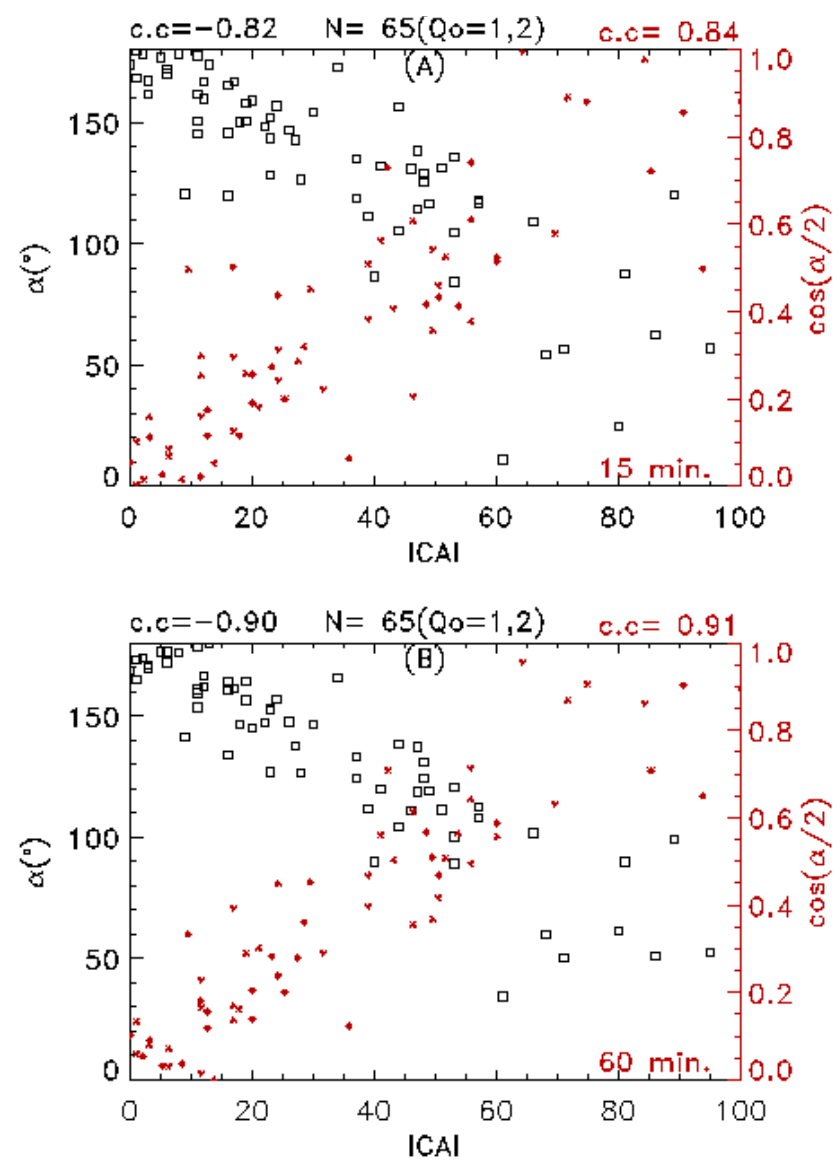

Fig. 3. The angle $\alpha$ (black squares) and $\cos (\alpha / 2)$ (red *) plotted against $|\mathrm{CA}|$ (in \%) all for $Q_{O}=1,2$ (where $N=65$ ), for the $15 \mathrm{~min}$ average cases in the top panel (A) and for the $1 \mathrm{~h}$ average cases in the bottom panel (B).

(from Eq. 1), both vs. $|\mathrm{CA}|$, for different MC-quality considerations, in testing Eq. (4). In all cases as we go to greater quality (and therefore to a smaller number of cases), we obtain larger correlation coefficients. As we also see, the c.c.s for $\alpha$ and $\cos (\alpha / 2)$ are almost the same for the same $Q_{O}$, but those for $\cos (\alpha / 2)$ are consistently better, as expected, or at least the same (for the $1 \mathrm{~h}$ interval, $N=23$ ). So in an average way the assumption of the circular cross-section is quite good and supports "parameter consistency," among $\alpha, R_{O}$ and $|\mathrm{CA}|$. Figure 3 shows the results of $\alpha$ and $\cos (\alpha / 2)$ plotted against $|\mathrm{CA}|$ (in the same panel), all for $Q_{O}=1,2$ (where $N=65$ ), for the $15 \mathrm{~min}$ average cases at the top (panel A) and for the $1 \mathrm{~h}$ average cases on the bottom (panel B). The $1 \mathrm{~h}$ set give the better c.c.'s for both $\alpha$ and $\cos (\alpha / 2)$, but slightly better for $\cos (\alpha / 2)$ (c.c. of 0.91), as expected.

In Table 2 we show the c.c.s for $\left\langle|\boldsymbol{B}|>,\langle|\boldsymbol{B}|>| B_{O}\right.$, and $B_{O}$, all vs. $|\mathrm{CA}|$. ( $B_{\mathrm{MAX}}$ vs. $|\mathrm{CA}|$ was also considered with very poor results across the board. This is not surprising, since anomalous peaks in $|\boldsymbol{B}|$, especially at the MC's front are not uncommon. Hence, examining $B_{\text {MAX }}$ is not useful in 
Table 1. Linear correlation coefficients for $\alpha$ and $\cos (\alpha / 2)$ vs. $|\mathrm{CA}|$.

\begin{tabular}{ccrrrr}
\hline N of events & $Q_{O}$ (and notes) & \multicolumn{2}{c}{15 min analysis interval } & \multicolumn{2}{c}{1 h analysis interval } \\
& & c.c. $\{\alpha\}$ & c.c. $\{\cos (\alpha / 2)\}$ & c.c. $\{\alpha\}$ & c.c. $\{\cos (\alpha / 2)\}$ \\
\hline 98 & All events & -0.71 & 0.74 & -0.76 & 0.80 \\
65 & 1 and 2 & -0.82 & 0.84 & -0.90 & 0.91 \\
23 & 1 only & -0.93 & 0.94 & -0.96 & 0.96 \\
\hline
\end{tabular}

Table 2. Linear correlation coefficients for $<|\boldsymbol{B}|>,<|B|>\mid B_{O}$ and $B_{O}$ vs. $|\mathrm{CA}|$.

\begin{tabular}{ccrrr}
\hline$N$ of events & $Q_{O}$ (and notes) & c.c. $\{<B>\}$ & c.c. $\left\{<B>\mid B_{O}\right\}$ & c.c. $\left\{B_{O}\right\}$ \\
\hline 98 & All events & -0.27 & -0.93 & 0.17 \\
65 & 1 and 2 & -0.28 & -0.88 & -0.02 \\
23 & 1 only & -0.37 & -0.92 & 0.00 \\
\hline
\end{tabular}

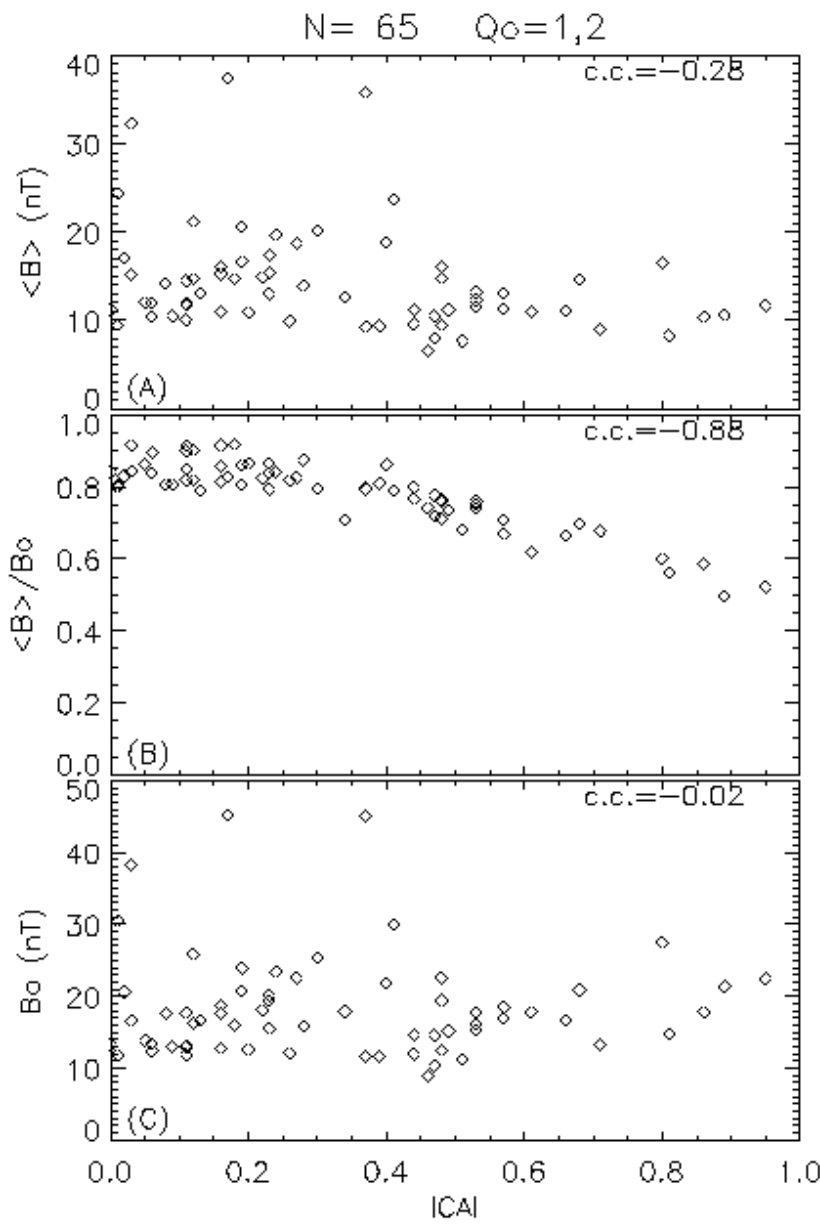

Fig. 4. $<|\boldsymbol{B}|>,<|\boldsymbol{B}|>\mid B_{O}$, and $B_{O}$, in panels (A), (B), and (C), respectively, each plotted against $|\mathrm{CA}|$ and all for $Q_{O}=1,2(N=65)$. The linear correlation coefficient (c.c.) for each quantity is shown in its panel. this type of analysis.) The c.c.s for $\langle|\boldsymbol{B}|>| B_{O}$ are all clustered near a good value of c.c. $=-0.90$ and, in fact, the one for "all events" $(N=98)$ is unusually good considering that $Q_{O}=3$ cases are included. As we also expect, the c.c.s for $B_{O}$ are either 0.00 or close to it. In fact, as we go to higher quality the $\mid$ c.c. $\mid$ for $B_{O}$ vs. $|\mathrm{CA}|$ gets lower, as it should, since there should be no correlation of $\mathrm{B}_{O}$ with $|\mathrm{CA}|$. The c.c.s for $<|\boldsymbol{B}|>$ vs. $|\mathrm{CA}|$ are poor, and this is also as expected. That is, without the normalization by $B_{O}$ we get weak correlation of $\langle|\boldsymbol{B}|>$ with $|\mathrm{CA}|$. The good correlation that we see with $<|\boldsymbol{B}|>\mid B_{O}$ is lost in examining only $<|\boldsymbol{B}|>$, no doubt because there is a deleterious mixing of effects between actual dropping off of field intensity with $|\mathrm{CA}|$ and independent (of $|\mathrm{CA}|$ ) variations of $\langle|\boldsymbol{B}|>$ due to differing solar birth conditions and possible solar cycle changes. The main point here is that relative field intensity $\left(<|\boldsymbol{B}|>\mid B_{O}\right)$ behaves as expected vs. $|\mathrm{CA}|$, on average, strengthening the "consistency argument."

Figure 4 shows the results of $\langle|\boldsymbol{B}|\rangle,\langle|\boldsymbol{B}|>| B_{O}$, and $B_{O}$ plotted against $|\mathrm{CA}|$ in panels (a), (b), and (c), respectively, all also for $Q_{O}=1,2$. We notice the tight clustering of $<|\boldsymbol{B}|>\mid B_{O}$ with respect to ordinate-values in panel (b). As expected, the c.c.s for $\left\langle|\boldsymbol{B}|>\right.$ (panel a) and $B_{O}$ (panel c) are very small and that for $\langle|\boldsymbol{B}|>| B_{O}$ (panel b) is quite large and significant. We fit a quadratic curve to the data in panel (b) (now for $\left[<|B|>\mid B_{O}\right]$ observations) and obtain the following:

$$
\begin{aligned}
{\left[<|B|>/ B_{O}\right] \mathrm{obs}^{\prime}=} & C_{O}+C_{1}|\mathrm{CA}|+C_{2}|\mathrm{CA}|^{2} \\
& (\text { for }|\mathrm{CA}|: 0.0 \text { to } 1.0)
\end{aligned}
$$

where $C_{O}=0.8567, C_{1}=-0.0304$ and $C_{2}=-0.3777$, with a small sigma for the fit of 0.0389 ; the prime on $\left[<|B|>/ B_{O}\right]$ obss $^{\prime}$ indicates that the observations have been fitted to the quadratic curve. This can be thought of as simply an overall quadratic smoothing of the data. In Fig. 5, panel (b) of Fig. 4 is again reproduced (i.e., $\left[<|B|>\mid B_{O}\right]$ 


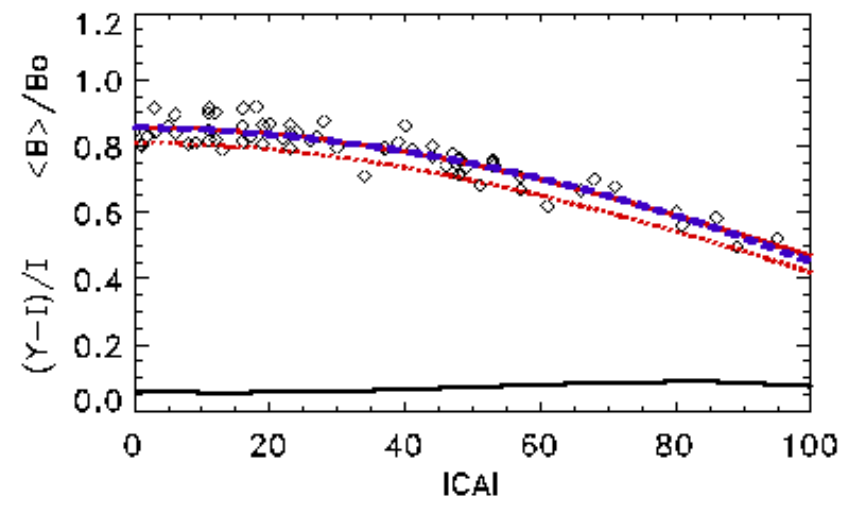

Fig. 5. Panel (B) of Fig. 4 showing $\left[<|B|>\mid B_{O}\right]$ (from observations) vs. $|\mathrm{CA}|$ (in \%), given by the black diamonds. The quadratic curve fitted version of this $(\equiv Y)$ is given by the dashed blue line (the primed quantity of Eq. 17). To this we superimpose the $\left[\left\langle|B|>/ B_{O}\right]\right.$ theory-curve (dotted red $\equiv I$ ), from Eq. (15); see text. The solid red curve is given by Eq. (20); this is the $\left[<|B|>\mid B_{O}\right]$ theory, mod estimate. The solid black curve at the bottom is given by $(Y-I) / I$; it is a relative change in $\left[<|B|>/ B_{O}\right]$ between observations and theory (before modification) - see Sect. 4 .

(from observations) vs. $|\mathrm{CA}|$ (in\%), as black diamonds), as well as its quadratic fit (dashed blue curve, the primed quantity of Eq. 17) shown, and the $\left[<|B|>\mid B_{O}\right]$ theory-curve (dotted red curve), from Eq. (15), is superimposed. It appears that, if Eq. (15) were multiplied by a constant $(S \approx 1.0)$ and slightly biased by constant " $a$," we would nearly obtain the dashed blue curve in Fig. 5. Hence,

$\left[<|B|>/ B_{O}\right]$ theory,mod $=S I+a$.

How do we find $S$ and $a$, and what do they mean? We now attack the fist question. In Fig. 6 we plot $\left[\langle|B|>| B_{O}\right]$ theory $(\equiv I)$ vs. $\left[<|B|>\mid B_{O}\right] \operatorname{obss}^{\prime}(\equiv Y)$; i.e., each point is for the same $|\mathrm{CA}|$. The result is nearly an exact straight line having a c.c. of 0.999 . Hence, a linear fit of this line gives

$Y \approx\left[<|B|>/ B_{O}\right]$ theory, $\bmod =S I+a$,

where we obtain $S=0.994$ and $a=+0.052$ from the fit, with a small sigma of 0.0028 . Hence, Eq. (18) becomes

$\left[<|B|>/ B_{O}\right]$ theory, $\bmod =0.994 I+0.052$,

which holds for all $|\mathrm{CA}|$. The quantity

$\left[<|B|>/ B_{O}\right]$ theory,mod from Eq. (20) is plotted in Fig. 5 as a solid red curve, and it very closely approximates the dashed blue curve in the figure which was a quadratic fit to the observed $\left[<|B|>/ B_{O}\right]$ obs $\left(\left[<|B|>/ B_{O}\right]\right.$ obs $\left.^{\prime}(\equiv Y)\right)$. But notice that to accomplish this good reproduction of observations with theory vs. $|\mathrm{CA}|$, we had to enhance the theoretical $<|B|>/ B_{O}$ curve by a slight bias of +0.052 (after first multiplying by $S=0.994)$. Even though " $a$ " is not exactly zero, and $S$ is not exactly 1.0, we have achieved remarkably good agreement between theory and observations, using our model

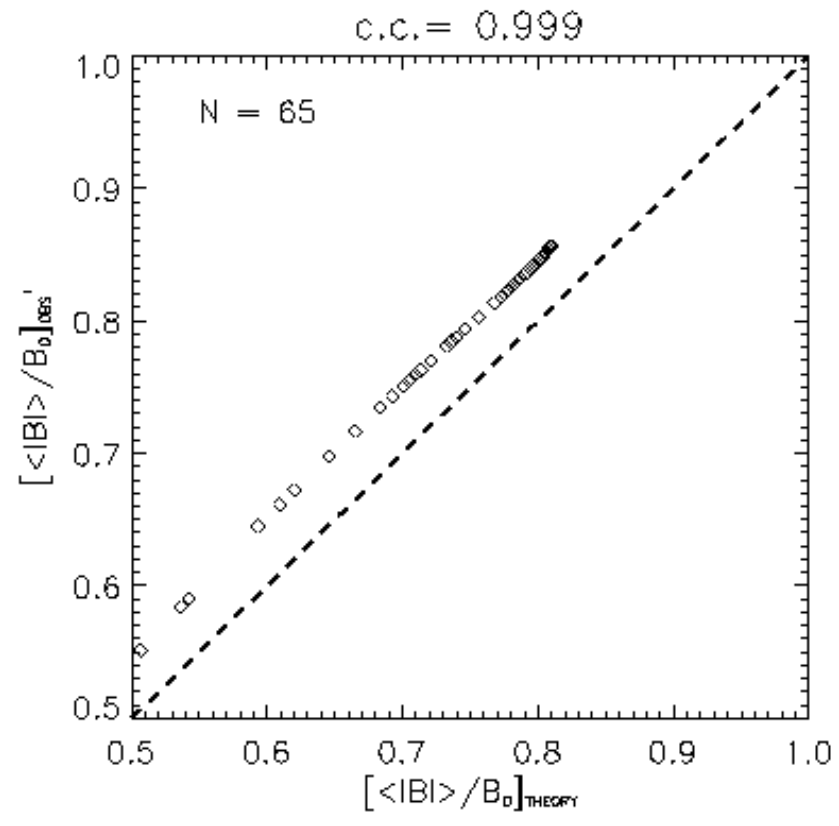

Fig. 6. $\left[<|B|>/ B_{O}\right]$ lobs' $^{\prime}$ (from the dashed blue curve of Fig. 5) vs. $\left[<|B|>\mid B_{O}\right]$ theory (from the dotted red curve of Fig. 5). These two sets are very well correlated, with c.c. $=0.999$. (This good correlation enables us to calculate $S$ and $a$ in Eqs. 18 and 19). The diagonal dashed line has a slope of exactly 1.0 and shows, by comparison, how closely the data follows this slope.

$|\mathrm{CA}| \mathrm{s}, B_{O}$ s, and derived coordinate system (through the values for $\theta_{A}$ and $\phi_{A}-$ see Sect. 3.1), suggesting again that we can have confidence in these estimated entities on average. But the differences between theory (before modification) and observations (as fitted by the quadratic) are worth investigating quantitatively, so we now form the normalize difference ratio

Ratio $\equiv(Y-I) / I$,

This Ratio is a measure of the relative change in

$\left[<|B|>\mid B_{O}\right]$ between fitted-observations and theory vs. $|\mathrm{CA}|$, and is given by the black curve in the bottom of Fig. 5 . Notice that this Ratio is relatively low and steady, suggesting that our relative measure of the effects of solar wind interaction and expansion on the structure of a MC (i.e., outside of the simple constant $\alpha^{\prime}$ force free solution to a MC's field structure) is, on average, weak and almost independent of the radial distance from the MC's axis. However, there is a modest increase in Ratio as $|\mathrm{CA}|$ increases, possibly suggesting that there is a greater influence of external compression in the outer reaches of the MC than in the inner regions, as one might expect. This finding is an interesting by-product of this analysis, but since there are many sources of uncertainly in its derivation, we should be cautious in our interpretation. 


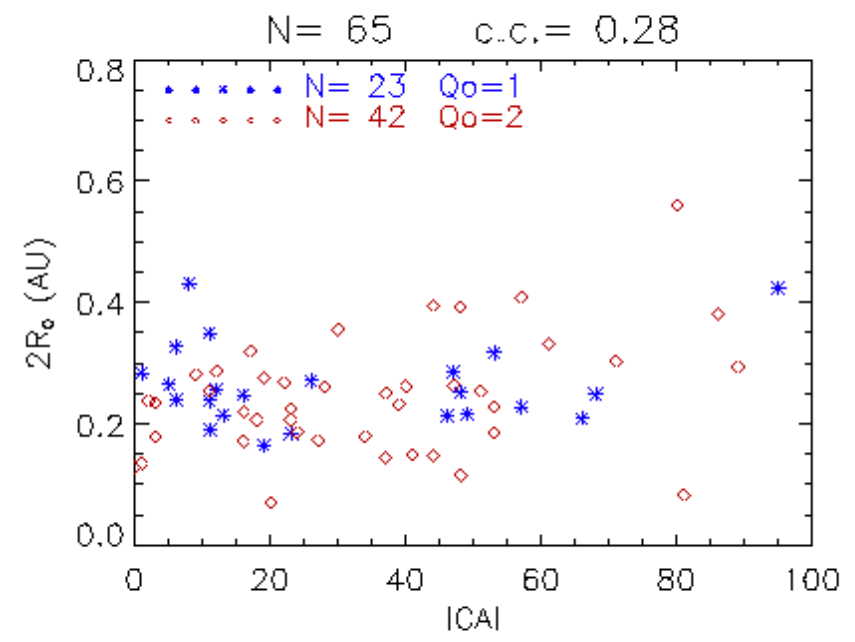

Fig. 7. A plot of the estimated diameters of the MCs $\left(2 R_{O}\right)$ vs. $|\mathrm{CA}|$ (in \%) for the combined $Q_{O}=1,2$ cases $(N=65)$, where c.c. $=0.28$, and where each quality-set is shown separately: for $Q_{O}=1 \quad(N=23$, by * in blue) and for $Q_{O}=2(N=42$, by diamonds in red).

\section{$5 \quad|\mathbf{C A}|$ vs. $\boldsymbol{R}_{\mathbf{O}}$}

In Fig. 7 we show a plot of the estimated diameter, $2 R_{O}$, vs. $|\mathrm{CA}|$ for the WIND MCs of quality sets $Q_{O}=1$ (blue *) and $Q_{O}=2$ (red diamonds). The c.c. is 0.28 for the two sets combined; separately the c.c.'s are $0.11\left(Q_{O}=1\right)$ and 0.38 $\left(Q_{O}=2\right)$, both being quite small. See Table 3 which compares the c.c.'s for $2 R_{O}$ vs. $|\mathrm{CA}|$ for various combinations of quality sets - again all showing small c.c.'s. It is evident that there is no significant linear correlation between the $|\mathrm{CA}| \mathrm{s}$ and the sizes of the MCs. So it is not likely that the MC sizes statistically control the sizes of $|\mathrm{CA}| \mathrm{s}$. It may be interesting to note, however, as also shown in Fig. 7, that for the $Q_{O}=1,2$ set of MCs the largest and smallest events tend to have $Q_{O}=2$ quality evaluations.

\section{Summary and conclusions}

WIND magnetic field data were used in this analysis covering the period from early 1995 until mid April 2006, and providing $N=98 \mathrm{MCs}$. Results are categorized according to the Quality $\left(Q_{O}\right)$ of the events considered. Appendix A of Lepping et al. (2006) gives the definition of $Q_{O}$. Most of the analysis was carried out using MCs of $Q_{O}=1$ and 2 combined (where $Q_{O}$ spans values of 1 (excellent), 2 (good), and 3 (poor)), i.e., the poor cases were not used for the main results, but some of their consequences were shown. We explained that we occasionally show estimated values of $|\mathrm{CA}| \mathrm{s}$ greater than $100 \%$, even though rarely are they actually so. In principle no value of $|\mathrm{CA}|$ for any realistic $\mathrm{MC}$ should be greater than $100 \%$, unless the MC is a core-annulus case (e.g., see Lepping et al., 2006), and they are uncommon; in this case the (inner) core's radius is at $R_{O}$. Even for the poorly fitted
Table 3. Linear correlation coefficients for $2 R_{O}$ vs. $|\mathrm{CA}|$.

\begin{tabular}{ccc}
\hline$N$ of events & $Q_{O}$ (and notes) & c.c. \\
\hline 98 & All events & 0.19 \\
65 & 1 and 2 & 0.28 \\
23 & 1 only & 0.11 \\
\hline
\end{tabular}

MCs (all of which are $Q_{O}=3$ cases), we choose to present the model's actual output estimate of $|\mathrm{CA}|$ rather than artificially assigning a value of $|\mathrm{CA}| \approx 100 \%$, even though the actual value must be near $100 \% \pm(E)$, where the error $E$ (i.e., $E$ with one sigma uncertainty) is usually quite big for many of the $Q_{O}=3$ cases. That it is even possible for model-estimated values of $|\mathrm{CA}|$ to be greater than $100 \%$ is due to the fact that the model does not constrain $Y_{O}$ to be equal to or less than $R_{O}$ (where recall that $|\mathrm{CA}|=\left|Y_{O}\right| / R_{O}$ ); they are both free parameters in the model. In summarizing a large early set of WIND MCs, Lepping et al. (2006, Fig. 13) show the typically large spreads of sigma $\{|\mathrm{CA}|\}$ s on MCs generally. In many cases, even when $Q_{O}$ is 1 or 2 (i.e., the good cases) the range of $E$ 's on $|\mathrm{CA}|$ is large, i.e., from $15 \%$ to $55 \%$, and for the $Q_{O}=3$ cases the $E$ 's on $|\mathrm{CA}|$ can range from $20 \%$ to $70 \%$, or even larger (but rarely). So, for example, if the estimated $|\mathrm{CA}|$ is $120 \pm 40 \%$, this surely allows for a $|\mathrm{CA}|$ smaller than $100 \%$ (i.e., within the range of $80 \%$ to $100 \%$ ), but we cannot give more information on where it is exactly in that range. In fact, we have previously shown that the $|\mathrm{CA}|$ parameter (i.e., $Y_{O}$ ) is usually the poorest determined of the full set of seven parameters that we fit with our model for any quality classification; see Lepping et al. $(2003,2004)$ on a study of the fit parameters for the force free model. In any case, the MC examples with poor parameter fittings $\left(Q_{O}=3\right)$ are of minor concern to us here, because we rely mainly on the $Q=1,2$ cases in the quantitative aspects of this study. It is precisely because of our concern about the usefulness and accuracy of estimated $|\mathrm{CA}|$ by our model, and $|\mathrm{CA}|$ 's important role in this study, that we emphasized the consistency arguments of Sect. 3.

Magnetic field observations within MCs indicate that as $|\mathrm{CA}|$ (in \%) approaches $100 \%$, both the magnitude and direction profiles of the field (vs. time) are typically somewhat flat, making it hard to identify a MC based only on magnetic field observations when there is a distant spacecraft passage. (However, examples of such flattening are not shown explicitly in this work.) This is true, of course, whether the MC is "within" an ICME or not. Also the success of our "consistency" arguments (Sects. 3 and 4 concerning both the $|\boldsymbol{B}|$ and $\alpha$-profiles) aids us in trusting sufficiently, and in an average way, the model-estimated quantities that were important to the analysis. These quantities are $R_{O},|\mathrm{CA}|, B_{O}$, and the MC-coordinate system in which the analysis was carried out. (Again we stress that the "consistency" arguments hold best for the $Q_{O}=1,2$ cases and only for a large number of events 
( $N=65$ ). For the $Q_{O}=3$ (poor) cases we should not always expect such satisfying results, as the correlation coefficients in Table 1 show for the $\alpha$ study, for example.) That being determined, we generate the distribution of the number of MCs vs. $|\mathrm{CA}|$ and showed in Fig. 1 how it decreases as $|\mathrm{CA}|$ increases, whether the event is ICME-related or not, where again a large number of cases are considered and for various quality subsets. Figure 1 shows that there is a dramatic drop off of the numbers of events as $|\mathrm{CA}|$ becomes larger for all subsets; Fig. A1 shows similar results where the MCs were parameterized according to high vs. low inclinations, split at $45^{\circ}$ (shown also to be true if split into three parts, i.e., at $30^{\circ}$ and $60^{\circ}$ ). In reality a complete distribution of $|\mathrm{CA}| \mathrm{s}$ may not be exactly uniform, but the estimated $|\mathrm{CA}| \mathrm{s}$ in Fig. 1 seem to have an unrealistically rapid drop-off, which we argue is due in part to a selection effect. By this we mean that it is usually more difficult to recognize a $\mathrm{MC}$ when the $|\mathrm{CA}|$ is large and where the magnitude of the field is relatively low and does not change very much in magnitude or direction over the duration of the spacecraft passage. In fact, it has been observed that $|\boldsymbol{B}|$ at large $|\mathrm{CA}|$ within a MC can drop to approximately ambient values especially in the rear of the MC (not shown in this work).

We first examined the field direction change from the inbound- to outbound-boundary within an average MC, through the " $\alpha$-analysis," and showed a relatively good correspondence between $\cos \alpha / 2$ vs. $|\mathrm{CA}|$ statistically, as expected ideally for our model (see Figs. 2 and 3). The correspondence was especially good when carried out using the 1 -h averages of the field which yielded a c.c. of 0.91 , as shown in the bottom panel of Fig. 3. We then concentrated on field intensity and normalized intensity.

In our attempt to theoretically reproduce (from the force free model) the actual average fall-off of the normalized magnetic field intensity in $\left[<|B|>\mid B_{O}\right]$ theory vs. $|\mathrm{CA}|$, we had to slightly decrease the theory-curve for

$\left[<|B|>/ B_{O}\right]$ theory by a factor of 0.994 (derived from $S$ ) and slightly bias it by $a=+0.052$; see Fig. 5 and the associated text. Although the values of $S$ and $a$ represent small adjustments, they are not insignificant. We are not sure that we fully understand their cause. However, they are likely, at least in part, the result of the fact that MCs interact with the solar wind which usually causes field compression within the MC, especially in the outer reaches. (But to be complete, the effects of MC expansion, especially in explaining the displaced location of $B_{\mathrm{MAX}}$ within a MC, for example, should be folded into this analysis also - see Farrugia et al. (1993). MC expansion occurs for most cases at $1 \mathrm{AU}$.) Field compression is often present in the upstream part of the MC but not so often in the downstream part. But the effect of the overall interaction (and expansion) is to cause an average intensity increase over the full duration, especially in the $\boldsymbol{Z}_{\mathrm{CL}}$-component of $\boldsymbol{B}$, and therefore also in $\langle|\boldsymbol{B}|\rangle$, without much change in the direction of the actual field from the simple force free field direction. And both effects (expansion and compression) usually tend to cause a very noticeable asymmetry in the $|\boldsymbol{B}|$-profile across the MC such that the field is usually stronger in its early part, and sometimes markedly so, at least at $1 \mathrm{AU}$. (But also at $1 \mathrm{AU}$ some relatively small number of MCs are quite different, i.e., some have relatively symmetric $|\boldsymbol{B}|$ s, peaking near the center, some $|\boldsymbol{B}|$ s are nearly flat, and some even peak somewhat late in the MC.) It is interesting to note that at greater distances from the Sun most MCs are seen to grow even larger according to a simple expansion rule, as seen in Voyager observations, for example (Burlaga and Behannon, 1982; Burlaga, 1995). However, even at distances of 2 to $4 \mathrm{AU}$ the field magnitude within a MC often retains some asymmetry in $|\boldsymbol{B}|$, but to a lesser extent than at $1 \mathrm{AU}$ (Burlaga and Behannon, 1982). Eventually, at much greater distances, the expansion is expected to significantly decrease or disappear and with it most of the asymmetry in $|\boldsymbol{B}|$ as the MC's magnetic field merges into the background interplanetary magnetic field. However, the details of this are not completely clear, so it is an area that needs further investigation

"Ratio" $(Y-I) / I$ (see Eq. 21) is a measure of the relative change in $\left[<|B|>\mid B_{O}\right]$ between observations and theory as a function of $|\mathrm{CA}|$, and its examination is another way of looking at the impact of $S$ not being exactly 1.0 and " $a$ " not being exactly 0.0 . Our analysis shows that Ratio is small and relatively steady with respect to small or medium sized $|\mathrm{CA}|$ s (i.e., up to $\sim 50 \%$ ), but it slowly increases as $|\mathrm{CA}|$ approaches $100 \%$, as shown by the black curve in Fig. 5 (bottom); actually something close to a plateau is reached. We interpret this to mean that the interaction/expansion effects on the structure of a MC are on average somewhat weak and almost independent of the radial distance from the MC's axis but are more noticeable in the outer reaches, due to external effects. Ratio's behavior is an interesting by-product of this study. (This behavior says nothing about $\left(<B|>| B_{O}\right)$ 's relationship to longitude within the MC, of course.) We stress that these results do not apply for any specific MC, where interaction/expansion effects may be strong and very noticeable, or very weak, depending on specific conditions.

Regardless of the well-known shortcomings arising from the simplicity of the Lepping et al. (1990) MC fitting model (force free of constant $\alpha^{\prime}$ ), we find that key fit-parameters are internally consistent on average especially for the $Q_{O}=1,2$ WIND set. And this set of MCs represents about $2 / 3$ of all cases know for that mission (i.e., up to and including the year 2006). Those key parameters are $R_{O}, B_{O}$, and CA, as well as the latitude $\left(\theta_{A}\right)$ and longitude $\left(\phi_{A}\right)$ of the MC's axis that provide the magnetic cloud coordinate system in which we carried out the analysis. And since handedness, another fit parameter in the model, is always known, we are accounting for the relatively good accuracy on average of 6 of the 7 fit parameters for the $Q_{O}=1,2$ WIND set. This is another byproduct of our analysis, and probably of greater importance than the one mentioned above. 
We suggest that a spacecraft passing through $\mathrm{MC}$ at a large $|\mathrm{CA}|$ can lead to the MC-encounter being overlooked, because MCs are much harder to identify when the $|\mathrm{CA}|$ is large. And this may happen frequently. However, this may not completely answer our original question (on the occurrences of ICMEs vs. MCs), and we must look for other factors, such as peculiarities of CME birth conditions. And a thorough analysis of the subject would require MC-solar source correlations. But such correlations are difficult and sometimes made all the more so, because some originally expelled MCs (seen at the Sun by any means) are expected to be missed by Earth (e.g., see Gopalswamy et al., 2008).

This point should be stressed: ICMEs are defined in terms of a far larger number of potential physical indicators than are used for MCs (e.g., see Schwenn, 1996), meaning perhaps 12 vs. 4 parameters, respectively, i.e., a ratio of 3 times as many for ICMEs than MCs. But different subsets of these 12 physical parameters are utilized for different ICMEs making it easier not to miss identifying an ICME encounter. And we point out that most of the ICME identifiers are independent of $|\mathrm{CA}|$, or at least weakly dependent on it, in contrast to the situation for MCs. This may be the principal cause of a poor MC-ICME correlation (e.g., Gosling, 1990, and Wu and Lepping, 2010), but specifically for the following reason. Suppose that a MC/CME structure is expelled from the Sun such that, as it passes a spacecraft at Earth, with say the encounter being far off the axis of the internal enhanced $|\boldsymbol{B}|$ portion of the overall helical field structure (indicating a poor or no MC encounter), but nevertheless with some of the 12 ICME-parameters being measured indicating the presence of an ICME. Many such ICME identifiers do not depend on a parameter such as $|\mathrm{CA}|$ (e.g., bi-directional streaming electrons), and more than a few ICME-indicators may be present. Hence, the MC-ICME correlation will be driven down for such cases. But, if the probability of observing both MCs and ICMEs depended on average $|\mathrm{CA}|$ in the same way, regardless of the particular value of $|\mathrm{CA}|$ for any case, correlation for a large set would be quite high, provided that the structures were highly correlated at the Sun in the first place, of course, which we do assume. This is a key element of our argument as to why, at least partly, an unusually low number of MCs are identified within ICMEs as observed at $1 \mathrm{AU}$ $(\approx 30 \%$ or smaller); see Gosling (1990) and Wu and Lepping (2010). And we speculate that if any magnetic structure even remotely possessing a MC field geometry and intensity were accepted as a real MC in the identification process, then the ratio of MCs-to-ICMEs would probably increase dramatically.

Russell and Shinde (2005) and Russell et al. (2005) discuss the well known problem of non-uniqueness associated with the identifications of ICMEs. They developed a new parameter to define ICMEs whereby the net plasma and field pressure perpendicular to the local magnetic field is used, with consideration of its difference from the ambient value, and even in their work several classes of events appear to
Table A1. Coefficients for Eq. (A1) ${ }^{\mathrm{a}}$.

\begin{tabular}{llrrr}
\hline Set considered & $N$ & $K_{O}$ & $b$ & $\sigma$ \\
\hline All $Q_{O}=1,2$ & 65 & 47.9 & $1.8 \times 10^{-6}$ & 40.7 \\
$0^{\circ} \leq\left|\theta_{A}\right|<45^{\circ}$ & 43 & 47.0 & $-8.6 \times 10^{-8}$ & 40.9 \\
$45^{\circ} \leq\left|\theta_{A}\right|<90^{\circ}$ & 22 & 49.1 & $8.1 \times 10^{-7}$ & 40.7 \\
\hline
\end{tabular}

a for $|\mathrm{CA}|$ measured in $\%$.

be possible. The Russell et al. (2005) approach to identifying ICMEs is perhaps the most satisfying yet in an attempt to avoid ambiguities. But, in any case, the broadness of the ICME's definition compared to that for MCs is very likely not the only factor in accounting for the lack of apparent coincidence of these two types of structures.

Finally, we see no significant correlation between the estimated $|\mathrm{CA}|$ values and the sizes of the MCs (measured by the ideally estimated diameter, $2 R_{O}$ by our model) for the WIND's $Q_{O}=1,2 \mathrm{MC}$ set; see Fig. 7. This was shown to be true also for the full set of $N=98 \mathrm{MCs}$, but displayed here for only the $Q_{O}=1,2$ set $(N=65)$. So, in general, it is unlikely that the distribution of sizes of MCs statistically controls the distribution of the observed sizes of $|\mathrm{CA}| \mathrm{s}$.

\section{Appendix A}

What should the distribution of $|\mathrm{CA}|$ really look like generally, if there were no selection effect? By using only observational data from a single spacecraft (or even from multiple spacecraft, if the number of them or their positions are insufficient) we may never know the answer to this question. However, it may be revealing to examine again the distribution of $|\mathrm{CA}| \mathrm{s}$ for the WIND set of MCs but now parameterized according to whether or not each MC is very inclined or not, representing a combination of birth conditions, birth locations, and resulting solar wind interactions. Hence, we choose to look at those cases of $\left|\theta_{A}\right|$ (absolute value of the estimated latitude of the axis of a MC) between $0^{\circ}$ and $45^{\circ}$ and those between $45^{\circ}$ and $90^{\circ}$, separately, where $\theta_{A}$ is strictly defined in Sect. 3.1. We show the results in Fig. A1. As in Fig. 1 (showing the observed distribution of $|\mathrm{CA}|$ ) these histograms show that there is a severe drop off of identified MCs as a function of $|\mathrm{CA}|$ for both sets, and they look similar. We try a least-squares fit to each of the three distributions (where the center point of each bar is the value fitted) to the normal distribution of variable amplitude, i.e., to

$N(x)=K_{O}(1+b x) \exp \left(-x^{2} / 2 \sigma^{2}\right)$

where $x \equiv|\mathrm{CA}|$ (in \%). We considered Eq. (A1) to be symmetric about $\mathrm{CA}=0$ and allowed both positive and negative CA for fitting purposes, providing 8 points to fit for each curve, with the understanding that only the positive CA domain is to be retained. $K_{O}, b, \sigma$ and the number $(N)$ of $\mathrm{MCs}$ 


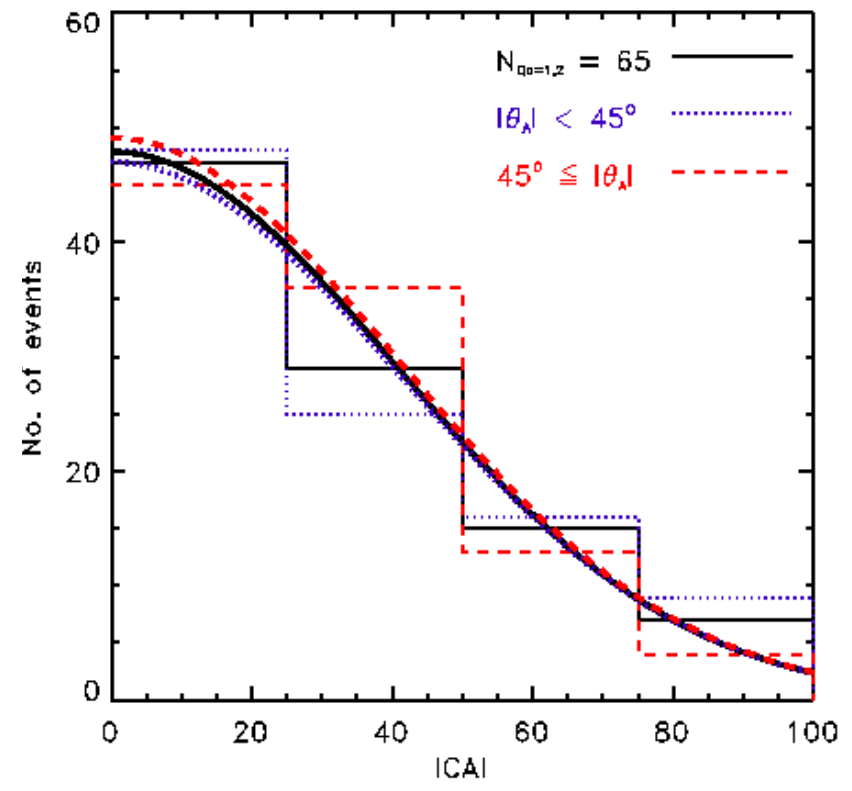

Fig. A1. Histograms of $|\mathrm{CA}|\left(\equiv\left|Y_{O}\right| / R_{O} \mid\right.$ (in \%)), for $Q_{O}=1$ and 2 combined split into those cases of $\left|\theta_{A}\right|$ between $0^{\circ}$ and $45^{\circ}$ and those between $45^{\circ}$ and $90^{\circ}$ separately, as well as for the full set of $N=65$ cases. Curves of normal distributions are least-squares fitted to all three distributions, showing very similar properties.

in each set are given in Table A1. Notice that $b$ is extremely small in each case and can be ignored, so the simple normal distribution is adequate for fitting these three distributions. We then see that all curves are essentially the same (within small errors) giving $K_{O} \approx 48$ and $\sigma \approx 41$. We carried out this same exercise with a split of three intervals of the $\left|\theta_{A}\right| \mathrm{s}$ according to $0^{\circ}$-to- $30^{\circ}, 30^{\circ}$-to- $60^{\circ}$, and $60^{\circ}$-to- $90^{\circ}$ and obtained essentially the same results as for the $0^{\circ}$-to- $45^{\circ}$ and $45^{\circ}$-to- $90^{\circ}$ split. So it does not matter if we split the distribution into low and high inclination MCs; we will get the same distribution in either case. We believe that this tells us that the specific inclinations of the MCs, regardless of their source, probably do not contribute to any discrepancy between the distributions of $|\mathrm{CA}|$ for MCs vs. ICMEs.

\section{Supplementary material related to this article is available online at: http://www.ann-geophys.net/28/1539/2010/ angeo-28-1539-2010-supplement.pdf.}

Acknowledgements. We thank the WIND/MFI and SWE teams, for the care they employ in producing the plasma and field data used for part of this work, and in particular we thank Keith Ogilvie, the SWE principal investigator, and Adam Szabo (PI) and Franco Mariani of the MFI team. We thank Daniel Berdichevsky for helpful comments. We are very grateful to the referee for pointing out an error in our original computation of the average model field across a magnetic cloud. This work was supported by the NASA Helio- physics Guest Investigator and Living With a Star programs under grant numbers NNG08EF51P and NNX07AH85G.

Topical Editor R. Forsyth thanks K. Marubashi for the help in evaluating this paper.

\section{References}

Bame, S. J., Asbridge, J. R., Feldman, W. C., Gosling, J. T., and Zwickl, R. D.: Bidirectional streaming of solar wind electrons $>80 \mathrm{eV}$ : ISEE evidence of a closed-field structure within the driver gas of an interplanetary shock, Geophys. Res. Lett., 8, 173-176, 1981.

Bothmer, V. and Schwenn, R.: Eruptive prominences as sources of magnetic clouds in the solar wind, Space Sci. Rev., 70, 215-220, 1994.

Bothmer, V. and Schwenn, R.: The structure and origin of magnetic clouds in the solar wind, Ann. Geophys., 16, 1-24, doi:10.1007/s00585-997-0001-x, 1998.

Burlaga, L. F.: Interplanetary Magnetohydrodynamics, Oxford Univ. Press, New York, 89-114, 1995.

Burlaga, L. F. and Behannon, K. W.: Magnetic clouds: Voyager observations between 2 and 4 AU, Solar Phys. 81, 181-192, 1982.

Burlaga, L. F., Sittler Jr., E. C., Mariani, F., and Schwenn, R.: Magnetic loop behind an inter- planetary shock: Voyager, Helios, and IMP-8 observations, J. Geophys. Res., 86, 6673-6684, 1981.

Burlaga, L. F., Klein, L., Sheeley Jr., N. R., Michels, D. J., Howard, R. A., Koomen, M. J., Schwenn, R., and Rosenbauer, H.: A magnetic cloud and a coronal mass ejection, Geophys. Res. Lett., 9(12), 1317-1320, 1982.

Burlaga, L., Fitzenreiter, R., Lepping, R., Ogilvie, K., Szabo, A., Lazarus, A, Steinberg, J., Gloeckler, G., Howard, R., Michels, D., Farrugia, C., Lin, R. P., and Larson, D. E.: A magnetic cloud containing prominence material: January 1997, J. Geophys. Res., 103, 277-285, 1998.

Burlaga, L. F., Ness, N. F., Richardson, J. D., and Lepping, R. P.: The Bastille Day Shock and Merged Interaction Region at 63 AU: Voyager 2 Observations, Solar Phys., 204, 399-410, 2001.

Burlaga, L. F., Lepping, R. P., and Jones, J. A.: Global configuration of a magnetic cloud, in: Physics of Magnetic Flux Ropes, Geophys. Monogr. Ser., vol. 58, edited by: Russell, C. T., Priest, E. R., and Lee, L. C., pp. 373-377, AGU, Washington, D.C., 1990.

Cane, H. V.: Coronal mass ejections and Forbush decreases, Space Sci. Rev., 93(1-2), 55-77, doi:10.1023/A:1026532125747, July 2000.

Cane, H. V. and Lario, D.: An introduction to CMEs and energetic particles, Space Sci. Rev., 123, 45-56, doi:10.1007/s11214-0069011-3, 2006.

Farrugia, C. J., Burlaga, L. F., Osherovich, V. A., Richardson, I. G., Freeman, M. P., Lepping, R. P., and Lazarus, A. J.: A study of an expanding interplanetary magnetic cloud and its interaction with the earth's magnetosphere: The interplanetary aspect, J. Geophys. Res., 98, 7621-7632, 1993.

Forbush, S. E.: On the effects in cosmic-ray intensity observed during the recent magnetic storm, Phys. Rev., 51, 1108-1109, 1937.

Galvin, A. B.: Minor ion composition in CME-related solar wind, in: Coronal Mass Ejections, Geophys. Monogr. Ser., vol. 99, edited by: Crooker, N., Joselyn, J., and Feynman, J., pp. 253260, AGU, Washington D.C., 1997. 
Gopalswamy, N., Hanaoka, Y., Kosugi, T., Lepping, R. P., Steinberg, J. T., Plunkett S., et al.: On the relationship between coronal mass ejections and magnetic clouds, Geophys. Res. Lett., 25, 2485-2488, 1998.

Gopalswamy, N.: Properties of interplanetary coronal mass ejections, Space Sci. Rev., 124, 145-168, doi:10.1007/s11214-0069102-1, 2006.

Gopalswamy, N., Akiyama, S., Yashiro, S., Michalek, G., and Lepping, R. P.: Solar sources and geospace consequences of interplanetary magnetic clouds observed during solar cycle 23 , J. Atmos. Solar-Terr. Phys., 70, 245-253, 2008.

Gosling, J. T.: Coronal mass ejections and magnetic flux ropes in interplanetary space, in: Physics of Magnetic Flux Ropes, Geophys. Monogr. Ser., vol. 58, edited by: Russell, C. T., Priest, E. R., and Lee, L. C., pp. 343-364, AGU, Washington, D.C., 1990.

Gosling, J. T.: Coronal mass ejections: An overview, in: Coronal Mass Ejections, Geophys. Monogr. Ser., vol. 99, edited by: Crooker, N., Joselyn, J., and Feynman, J., pp. 9-16, AGU, Washington D.C., 1997.

Gosling, J. T., Pizzo, V., and Bame, S. J.: Anomalously low proton temperatures in the solar wind following interplanetary shock waves : Evidence of magnetic bottles?, J. Geophys. Res., 78, 2001-2009, 1973.

Gosling, J. T., Bame, S. J., McComas, D. J., and Phillips, J. L.: Coronal mass ejections and large geomagnetic storms, Geophys. Res. Lett., 17, 901-904, 1990.

Henke, T., Woch, J., Mall, U., Livi, S., Wilken, B., Schwenn, R., Gloeckler, G., von Steiger, R., Forsyth, R. J., and Balogh, A.: Differences in the $\mathrm{O}^{7+} / \mathrm{O}^{6+}$ ratio of magnetic cloud and noncloud Coronal Mass Ejections, Geophys. Res. Lett., 25, 34653468, 1998.

Henke, T., Woch, J., Schwenn, R., Mall, U., Gloeckler, G., von Steiger, R., Forsyth, R. J., and Balogh, A.: Ionization state and magnetic topology of coronal mass ejections, J. Geophys. Res., 106, 10597-10613, 2001.

Klein, L. and Burlaga, L. F.: Interplanetary magnetic clouds at 1 AU, J. Geophys. Res., 87, 613-624, 1982.

Kahler, S. W.: Origin and properties of solar energetic particles in space, in: Space Weather, Geophys. Monogr. Ser., vol. 125, edited by: Song, P., Singer, S. J., and Siscoe, G. L., pp. 109-122, AGU, Washington D.C., 2001.

Kumar, A. and Rust, D. M.: Interplanetary magnetic clouds, helicity conservation, and current-core flux ropes, J. Geophys. Res., 101, 15667-15684, 1996.

Lee, M. A. and Fisk, L. A.: Shock acceleration of energetic particles in the heliosphere, Space Sci. Rev., 32, 205-228, 1982.

Lepping, R. P., Jones, J. A., and Burlaga, L. F.: Magnetic field structure of interplanetary magnetic clouds at 1 AU, J. Geophys. Res., 95, 11957-11965, 1990.

Lepping, R. P., Acuna, M. H., Burlaga, L. F., et al.: The WIND magnetic field investigation, The Global Geospace Mission, Space Sci. Rev., 71, 207-229, 1995.

Lepping, R. P., Berdichevsky, D. B., and Ferguson, T.: Estimated errors in magnetic cloud model fit-parameters with force free cylindrically symmetric assumptions, J. Geophys. Res., 108(A10), 1356, doi:10.1029/2002JA009657, 2003.

Lepping, R. P., Berdichevsky, D. B., and Ferguson, T.: Correction (to paper entitled Estimated errors in magnetic cloud model fit-parameters with force free cylindrically symmetric assump- tions by the same authors), J. Geophys. Res., 109, A07101, doi:10.1029/2004JA010517, 2004.

Lepping, R. P., Wu, C.-C., and Berdichevsky, D. B.: Automatic identification of magnetic clouds and cloud-like regions at $1 \mathrm{AU}$ : occurrence rate and other properties, Ann. Geophys., 23, 26872704, doi:10.5194/angeo-23-2687-2005, 2005.

Lepping, R. P., Berdichevsky, D. B., Wu, C.-C., Szabo, A., Narock, T., Mariani, F., Lazarus, A. J., and Quivers, A. J.: A summary of WIND magnetic clouds for years 1995-2003: model-fitted parameters, associated errors and classifications, Ann. Geophys., 24, 215-245, doi:10.5194/angeo-24-215-2006, 2006.

Luhmann, J. G.: CMEs and Space Weather, in: Coronal Mass Ejections, Geophys. Monogr. Ser., vol. 99, edited by: Crooker, N., Joselyn, J., and Feynman, J., pp. 291-299, AGU, Washington D.C., 1997.

Marsden, R. G., Sanderson, T. R., Tranquille, C., Wenzel, K.-P., and Smith, E. J.: ISEE 3 observations of low-energy proton bidirectional streaming events and their relation to isolated interplanetary magnetic structures, J. Geophys. Res., 92, 11009-11019, 1987.

Marubashi, K.: Structure of the interplanetary magnetic clouds and their solar origins, Adv. Space Res., 6(6), 335-338, 1986.

Marubashi, K.: Interplanetary magnetic flux ropes and solar filaments, in: Coronal Mass Ejections, Geophys. Monogr. Ser., vol. 99, edited by: Crooker, N., Joselyn, J., and Feynman, J., pp. 147156, AGU, Washington D.C., 1997.

McComas, D. J., Gosling, J. T., Winterhalter, D., and Smith, E. J.: Interplanetary magnetic field draping about fast coronal mass ejecta in the outer heliosphere, J. Geophys. Res., 93, 2519-2526, 1988.

McPherron, R. L.: Magnetospheric dynamics, in: Introduction to Space Physics, edited by: Kivelson, M. G. and Russell, C. T., Cambridge University Press, New York, 1995.

Ogilvie, K. W., Chornay, D. J., Fritzenreiter, R. J., et al.: SWE, A comprehensive plasma instrument for the WIND spacecraft, The Global Geospace Mission, Space Sci. Rev., 71, 55-77, 1995.

Priest, E.: The equilibrium of magnetic flux ropes (Tutorial lecture), in: Physics of Magnetic Flux Ropes, Geophys. Monogr. Ser., vol. 58, edited by: Russell, C. T., Priest, E. R., and Lee, L. C., pp. 122, AGU, Washington D.C., 1990.

Richardson, I. G., Dvornikov, V. M., Sdobnov, V. E, and Cane, H.: Bi-directional particle flows at cosmic ray and lower $(\sim 1 \mathrm{MeV})$ energies and their association with interplanetary coronal mass ejections/ejecta, J. Geophys. Res., 105, 12579-12591, 2000.

Richardson, I. G. and Cane, H.: The fraction of interplanetary coronal mass ejections that are in magnetic clouds: Evidence for a solar cycle variation, Geophys. Res. Lett., 31, L18804, doi:10.1029/2004GL020958, 2004.

Richardson I. G. and Cane, H. V.: Near-Earth interplanetary coronal mass ejections during solar cycle 23 (1996-2009): Catalog and summary of properties, Solar Phys., 264, 189-237, doi:10.1007/s11207-010-9568-6, 2010.

Russell, C. T. and Shinde, A. A.: What defines an interplanetary coronal mass ejection?, Solar Phys., 229, 323-344, 2005.

Russell, C. T., Shinde, A. A., and Jian, L.: A new parameter to define interplanetary coronal mass ejections, Adv. Space Res., 35, 2178-2184, 2005.

Schwenn, R.: Mass ejections from the sun and their interplanetary counterparts, Solar Wind 8, edited by: Winterhalter, D., Gosling, 
J. T., Habbal, S. R., Kurth, W. S., and Neugebauer, M., 426-429, 1996.

Schwenn, R., Rosenbauer, H., and Muhlhauser, K. H.: Singlyionized helium in the driver gas of an interplanetary shock wave, Geophys. Res. Lett., 7, 201-204, 1980.

Skoug, R. M., Feldman, W. C., Gosling, J. T., McComas, D. J., Reisenfeld, D. B., Smith, C. W., Lepping, R. P., and Balogh, A.: Radial variation of solar wind electrons inside a magnetic cloud observed at 1 and 5 AU, J. Geophys. Res., 105, 27269-27275, 2000.

Webb, D. F., Clive, E. W., Crooker, N. U., St. Cyr, O. C., and Thompson, B. J.: Relationship of halo coronal mass ejections, magnetic clouds, and magnetic storms, J. Geophys. Res., 105, 7491-7508, 2000.

Webb, D. F., Crooker, N. U., Plunkett, S. P., and St. Cyr, O.C.: The solar sources of geoeffective structures, in: Space Weather, Geophys. Monogr. Ser., vol. 125, edited by: Song, P., Singer, S. J., and Siscoe, G. L., pp. 123-141, AGU, Washington D.C., 2001.
Wu, C.-C., Lepping, R. P., and Gopalswamy, N.: Relationships among magnetic clouds, CMEs and geomagnetic storms, Solar Phys., 239, 440-460, 2006.

Wu, C.-C. and Lepping, R. P.: Comparison of the characteristics of magnetic clouds with those of interplanetary coronal mass ejections for solar cycle 23, Solar Phys., submitted, 2010.

Xu, D., Chen, T., Zhang, X. X., and Liu, Z.: Statistical relationship between solar wind conditions and geomagnetic storms in 19982008, Planet. and Space Sci., 57, 1500-1513, 2009.

Zwickl, R. D., Asbridge, J. R., Bame, S. J., Feldman, W. C., Gosling, J. T.: $\mathrm{He}^{+}$and other unusual ions in the solar wind: A systematic search covering 1972-1980, J. Geophys. Res, 87, 7379-7388, 1982. 